Steel, R. \& WALker, T. K. (1957). J. gen. Microbiol. 17, 445-452

\title{
A Comparative Study of Cellulose-Producing Cultures and Celluloseless Mutants of certain Acetobacter Spp.
}

\author{
By R. STEEL AND T. K. WALKER \\ College of Science and Technology, Manchester
}

SUMMARY: Comparative studies have been conducted with two strains of Acetobacter acetigenum (NCIB 8132, NCIB 5346), one strain of $A$. xylinum var. africanum (NCIB 7029) and a total of 26 celluloseless mutants obtained from these three parent cultures. In addition to the loss of ability to produce a cellulose pellicle, other consistent differences were also observed between the parent and the mutant cultures. Whereas the parent cultures oxidized ethanol to acetic acid this reaction was not observed with mutant organisms. The mutants showed (1) optimum growth in alkaline media, (2) growth in glucose yeast-extract medium in the presence of $10-12 \%(v / v)$ ethanol, (3) proteolytic activity, (4) growth at $40^{\circ}$. and (5) growth in yeast-extract medium; these abilities were not shown by the wild-type cultures. Variations in colony form, nutritional requirements, the ability to produce acid from certain sugars and to oxidize glycerol appeared to occur at random among the mutant cultures.

In their paper about 'celluloseless mutants' of Acetobacter xylinum, Schramm \& Hestrin (1954) did not mention any other differences between these mutants and the wild-type cellulose-producing culture. On the other hand, the cultures of 'celluloseless' mutants examined by Steel \& Walker (1957) differed from the wild-type cultures in colony form and in growth rate on glucose yeastextract agar medium. The present work was undertaken to determine other differences or similarities between the cellulose-producing organisms and the celluloseless mutants obtained from them.

\section{METHODS}

Cultures studied and cultural conditions. The cellulose-producing cultures which were examined were two strains of Acetobacter acetigenum (NCIB 8132 and NCIB 5346) and one strain of $A$. xylinum var. africanum (NCIB 7029) obtained from the National Collection of Industrial Bacteria, Teddington, Middlesex. Cultures of celluloseless mutants were obtained from these three parent cultures by methods described previously (Steel \& Walker, 1957). In all, a total of 26 mutant cultures was obtained by eight different methods (Table 1). Six of the mutant isolates were obtained from organism NCIB 8132 when it lost the ability to produce a cellulose pellicle during the course of the present work. Three of the mutant cultures died during the present study; apparently the cultures should be transferred at weekly intervals to ensure their viability. One other culture reverted to cellulose-formation after it had failed to produce cellulose pellicles in 4 subcultures ( 2 weeks).

Unless otherwise stated, all studies were made according to procedures 
outlined by Conn (1949). Glucose yeast-extract (GYE) medium ( $\mathrm{pH} \mathrm{7.0)}$ contained glucose $3 \%(\mathrm{w} / \mathrm{v})$ and Difco yeast-extract $0.5 \%(\mathrm{w} / \mathrm{v})$ in distilled water; for solid media, agar $(2 \%, w / v)$ was added. Malt wort medium was prepared by the method of Tosic \& Walker (1946). The parent cultures were kept in malt wort medium since certain cultures lost their ability to produce a cellulose pellicle when grown continuously in GYE medium (Steel \& Walker, 1957). The mutant organisms were stored on GYE medium because certain of these cultures failed to grow in malt wort medium (see later). With the exceptions mentioned later, media were sterilized in $8 \mathrm{ml}$. amounts in $18 \times 155 \mathrm{~mm}$. test tubes for $10 \mathrm{~min}$. at $15 \mathrm{lb}$./sq.in. pressure; inoculations were made by wire loop from $48 \mathrm{hr}$. cultures in GYE medium and incubation was at $30^{\circ}$ unless otherwise stated.

\section{Table 1. Sources of mutant cultures of Acetobacter spp. studied}

No. of mutant cultures

Method by which obtained*

Glucose yeast-extract (GYE) medium, $\mathrm{SH} \dagger$ Ethanol yeast-extract (EYE) medium, SH $\ddagger$ Selective medium + ethanol, ST\$

Ethyleneglycol + yeast-extract medium, ST EYE medium, ST $\ddagger$

GYE medium, mass plating

Selective medium, replica plating

GYE medium, ST

GYE medium, ST

GYE medium, ST
Parent culture

A. acetigenum NCIB 8132

A. acetigenum NCIB 8132

A. acetigenum NCIB 8132

A. acetigenum NCIB 8132

A. acetigenum NCIB 8132

$A$. acetigenum NCIB 8132

A. acetigenum NCIB 8132

$A$. acetigenum NCIB 8132

$A$. acetigenum NCIB 5346

$A$. xylinum var. africanum NCIB 7029

Total selected for study

5

3

1

1

1

2

1

6

$\mathbf{3}$

$\mathbf{3}$

26

* Indicates the conditions under which the parent culture was grown (cf. Steel \& Walker, 1957).

$\dagger$ SH $=$ shaken culture.

$\ddagger$ EYE medium containing ethanol $(20 \%, \mathrm{v} / \mathrm{v})$.

$\S \mathrm{ST}=$ static culture.

Frateur's tests. The 'Oxydogramme' tests recommended by Frateur (1950) for the classification of Acetobacter species were followed. Briefly, washed organisms (washed pellicles in the cases of the parent cultures) obtained from $48 \mathrm{hr}$. GYE cultures were incubated on the surface of sterile agar media containing the desired substrate $(2 \%, \mathrm{w} / \mathrm{v}$ or $\mathrm{v} / \mathrm{v}$, for solids and liquids, respectively). Acid production from ethanol and from glucose was detected by the dissolution of calcium carbonate $(2 \%, \mathrm{w} / \mathrm{v})$ present in the medium. Subsequent oxidation of the acetic acid produced from ethanol was shown by the reprecipitation of calcium carbonate on further incubation. Oxidation of calcium lactate to acetate is shown by the formation of a clear zone surrounding the added bacteria. The oxidation of glycerol to dihydroxyacetone was observed by the precipitation of copper oxide when the plates were flooded with Fehling's solution. Catalase was detected by the evolution of oxygen when Petri dishes containing the desired culture (GYE medium) were flooded with $2 \%(\mathrm{v} / \mathrm{v})$ hydrogen peroxide (100 vol.) in distilled water. 
Nutritional studies. The quantities $(\%, w / v$, in distilled water) of various ingredients used in defined media were as follows: glucose, $3.0 ;\left(\mathrm{NH}_{4}\right)_{2} \mathrm{SO}_{4}$, $0.3 ; \mathrm{KH}_{2} \mathrm{PO}_{4}, 0 \cdot 3 ; \mathrm{MgSO}_{4} \cdot \mathrm{7H}_{2} \mathrm{O}, 0.2$; Casamino acids (Difco), 1.0; biotin, 0.00003; riboflavin, 0.0002; calcium pantothenate, 0.0002. These vitamins were used previously by Creedy, Jowett \& Walker (1954) for the cultivation of Acetobacter acetigenum. The media ( $\mathrm{pH} \mathrm{5.9)}$ ) were sterilized in $6 \mathrm{ml}$. amounts in nephelometer tubes. Mutant organisms were transferred from $48 \mathrm{hr}$. GYE agar-slope cultures to $10 \mathrm{ml}$. sterile distilled water and one drop of this suspension (c. 0.05 ml.) was used as inoculum for each tube of test medium. In the case of parent cultures, a $48 \mathrm{hr}$. pellicle, washed with four changes of sterile distilled water, was shaken in the test medium as an inoculum. Each culture was tested in duplicate. Growth was assessed by measurement of the turbidity with a nephelometer or, in the case of cellulose-producing cultures, by visual assessment. The cultures were incubated for 7 days.

Production of acid and gas from sugars. The basal medium ( $\mathrm{pH} \mathrm{7 \cdot 0)}$ consisted of yeast-extract (Difco, $0.5 \%, w / v)+$ bromcresol purple $(0.003 \%, w / v)$ in distilled water; the test sugars were added to $1 \%(w / v)$. Each tube of medium contained an inverted Durham tube to test for gas production. The cultures were examined daily for 8 days.

Optimum $\mathrm{pH}$ range for growth. Growth was assessed after 3 days' incubation in GYE medium at initial $\mathrm{pH}$ values ranging from 4.0 to 9.0 in increments of $0.5 \mathrm{pH}$ units. Also, growth curves of certain mutant cultures (initial $\mathrm{pH} 7 \cdot 0$ ) were obtained by measurement of the turbidity with a nephelometer.

Tolerance to ethanol. These tests were made in GYE medium containing from 2 to $14 \%(\mathrm{v} / \mathrm{v})$ ethanol $(95 \%)$ in increments of $2 \%$; sterile ethanol was added aseptically to sterile GYE medium.

Growth at $40^{\circ}$. After inoculation into GYE medium, the cultures were incubated at $40^{\circ}$ for 5 days.

Growth in milk. A sample of skim milk (pH 6.5) was sterilized with free steam for $30 \mathrm{~min}$. on 3 consecutive days before inoculation with the test organisms; incubation was for 14 days.

Liquefaction of gelatin. The test medium was gelatin $(12 \%, w / v)+$ yeast extract (Difco, $0.5 \%$, w/v) in distilled water. Organisms from GYE agar slant cultures (4 days) were used as inoculum; incubation was at room temperature $\left(c .18^{\circ}\right)$ for 4 weeks.

Production of ammonia and hydrogen sulphide. The growth medium (pH 7•0) was Evans's peptone $(2 \%, \mathrm{w} / \mathrm{v})+$ yeast extract (Difco, $0.5 \%, \mathrm{w} / \mathrm{v})$ in distilled water. After 3 days of incubation the cultures were boiled in the presence of litmus paper to detect ammonia; hydrogen sulphide was detected by the method of Morse \& Weaver (see Conn, 1949).

Production of indole and reduction of nitrate. The test media (aqueous) contained peptone $(2 \%, \mathrm{w} / \mathrm{v})$, yeast extract $(0.5 \%, \mathrm{w} / \mathrm{v})$ and either casein (fat free, Hopkin and Williams Ltd.) $(0.5 \%, w / v)$ or potassium nitrate $(0.1 \%, w / v)$ respectively, depending whether the test was to determine indole production or nitrate reduction. The cultures were tested at 5 days. 


\section{RESULTS}

During the present work no marked differences were found between the different species of wild-type organisms examined; in fact, there was as much variability in properties between the two different strains examined as there was between the two different species (see Table 4). Also, the mutant organisms obtained from the different strains and species of wild-type cultures were indistinguishable. For these reasons the results are presented collectively.

Description of colonies. On GYE agar medium colonies of organisms NCIB 8132, NCIB 5346 and NCIB 7029 were punctiform, $1 \mathrm{~mm}$. or less in diameter, pale brown in colour, convex, with a smooth semi-matt to shiny surface, regular edge, and tough consistency; the whole colony lifted off the agar when touched with a needle; the colonies appeared on the plates after 3-5 days of incubation (Pl. 1, fig. 1). At $24 \mathrm{hr}$. colonies of all mutant cultures were $2-4 \mathrm{~mm}$. in diameter, white, circular, flat, with a diffuse edge, butyrous consistency, smooth and semi-matt surface showing lighter and darker areas (Pl. 1, fig. 2). At $48 \mathrm{hr}$. the colony form of 21 of the 26 cultures had changed to that shown in Pl. 1, fig. 3, which afforded the following description: colonies $3-5 \mathrm{~mm}$. in diameter, circular, white to cream-coloured, raised, regular edge, smooth and glistening surface, butyrous consistency. Three of these mutant cultures sometimes showed a different colony form after 4-6 days of incubation (Pl. 1, fig. 4). The five remaining cultures gave colonies at $48 \mathrm{hr}$. which were $2-5 \mathrm{~mm}$. in diameter, white, circular, convex, with a semi-matt surface and butyrous consistency; secondary colonies were produced by two of these cultures after 5-7 days' incubation (Pl. 1, figs. 5, 6). Yellow pigmentation was produced by one mutant culture, and three others showed an area in the centre of the colony suggestive of autolysis. Both of these latter characteristics only appeared in old cultures (10-14 days) and were variable.

Morphology, size, staining reaction and motility. Vegetative organisms of parent and mutant cultures were Gram-negative motile rods when cultivated for $24 \mathrm{hr}$. at $30^{\circ}$ in GYE medium; involution forms were also present in most cultures. All isolates examined were aerobic. The majority of mutant organisms were within the range $0 \cdot 4-0.8 \mu$. wide $\times 0 \cdot 6-2 \cdot 0 \mu$. long when stained with methylene blue. Spore formation was not observed with any of the isolates studied. Capsule formation was observed with the parent cultures when grown for $24 \mathrm{hr}$. in GYE liquid medium, whereas only some of the mutant isolates produced a capsule when grown under these conditions. When grown for $24 \mathrm{hr}$. on GYE agar medium, all mutants showed capsules.

Growth in GYE medium. Growth in GYE medium was similar to that described previously (Steel \& Walker, 1957). Briefly, parent cultures produced a surface pellicle of cellulose within $48 \mathrm{hr}$. incubation and the medium under the pellicle remained virtually clear; in contrast, the mutants grew diffusely throughout the medium within $24 \mathrm{hr}$. of incubation and did not produce a cellulose pellicle. Two of the mutant cultures generally showed abundant 'rope' formation. Growth curves of 9 out of 11 mutant isolates examined on GYE medium were of the diauxic type (Monod, 1947). 
Growth in malt wort medium. Of 7 mutant isolates tested, 3 did not grow in malt wort medium; the 3 parent cultures grew well.

Growth on GYE agar slopes. At 3-5 days of incubation, growth of the parent cultures was of the beaded type. At $24 \mathrm{hr}$. of incubation growth of mutant cultures was moderate, white, filiform, glistening and butyrous. Differences in growth type between the various mutant isolates were very slight. After prolonged incubation (10 days) eleven of the mutant isolates produced a yellow pigment; this, however, was a variable property.

Frateur's tests. A summary of the results obtained by using the tests recommended by Frateur (1950) is given in Table 2. All parent and mutant isolates produced catalase but did not oxidize calcium lactate. The parent cultures oxidized ethanol, acetate, glycerol (ketogenic activity) and glucose. In contrast, the mutant isolates showed no evidence of acid production from ethanol or glucose, while ketogenic activity was shown only by certain of these cultures. To obtain a positive reaction in the latter test it was necessary to incubate mutant organisms with substrate for 24-48 hr. before adding Fehling's solution; the recommended incubation period (Frateur, 1950) of 1-2 hr. gave positive results with parent cultures only. The positive reactions given by the majority of the mutant isolates were very weak in comparison with those given by the parent cultures. In other experiments with a Warburg respirometer (Umbreit, Burris \& Stauffer, 1945), suspensions of two mutant isolates examined showed no oxygen uptake.with ethanol as substrate whereas oxygen consumption with glucose was quite rapid.

Table 2. Proportion of cultures tested which gave a positive reaction with Frateur's tests (Oxydogramme method)

\begin{tabular}{lcc} 
& \multicolumn{2}{c}{ Cultures } \\
\cline { 2 - 3 } Parent & Mutant \\
Oxidase present & $3 / 3$ & $26 / 26$ \\
Oxidation of calcium lactate & $0 / 3$ & $0 / 26$ \\
Oxidation of glycerol to dihydroxyacetone & $3 / 3$ & $0 / 26$ \\
Oxidation of glucose to gluconic acid & $3 / 3$ & $14 / 26$ \\
& $3 / 3$ & $0 / 26$
\end{tabular}

Nutritional studies. A summary of these findings is given in Table 3. The parent cultures grew in glucose + salts medium and at a faster rate when vitamins were added; however, they did not grow in the Casamino acids + salts medium. The nutritional requirements were variable among the mutant isolates; 16 of 21 isolates showed either poor or moderate growth in glucose + salts medium and, in general, the same isolates showed a similar amount of growth when vitamins were added. Casamino acids + salts medium supported the growth of 17 of 21 mutant cultures, but the addition of glucose to this medium did not appreciably affect the amount of growth obtained. Only one mutant isolate did not grow in the medium containing all components; this culture grew moderately well in GYE medium. 
Table 3. Proportion of cultures which grew in certain defined media

\begin{tabular}{lcc}
\multicolumn{1}{c}{ Medium } & Parent & Mutant \\
Glucose, salts & $\mathbf{3} / \mathbf{3}$ & $16 / 21$ \\
Casamino acids, salts & $\mathbf{0} / \mathbf{3}$ & $\mathbf{1 7 / 2 1}$ \\
Glucose, salts, vitamins & $\mathbf{3} / 3$ & $17 / 21$ \\
Glucose, salts, Casamino acids & $\mathbf{3} / \mathbf{3}$ & $19 / 21$ \\
Glucose, salts, vitamins, Casamino acids & $\mathbf{3} / 3$ & $20 / 21$
\end{tabular}

Production of acid and gas from sugars. It may be noted from the results (Table 4) that acid was produced from more sugars by the mutant than by the parent cultures; however, acid was not produced by all of the mutant cultures from any one sugar. Lactose was the only sugar from which acid was not produced by any mutant. It is noteworthy that certain mutant isolates which had given a negative result by Frateur's method (Table 2) produced acid from glucose by this method of test. Gas production was not observed.

Table 4. Proportion of cultures tested which produced acid from certain sugars

\begin{tabular}{lcc} 
& \multicolumn{2}{c}{ Cultures } \\
\cline { 2 - 2 } Parent & Mutant \\
Xylose & $2 / 3^{*}$ & $8 / 18$ \\
Glucose & $1 / 3 \dagger$ & $9 / 18$ \\
Fructose & $3 / 3$ & $14 / 18$ \\
Galactose & $0 / 3$ & $14 / 18$ \\
Lactose & $0 / 3$ & $8 / 18$ \\
Sucrose & $0 / 3$ & $0 / 18$ \\
Maltose & $0 / 3$ & $14 / 18$ \\
A & $0 / 3$ & $2 / 18$
\end{tabular}

* Acid produced by cultures NCIB 8132 and NCIB 7029.

$\dagger$ Acid produced by culture NCIB 8132.

Table 5. Reaction of parent and of mutant cultures to certain tests

Optimum pH range for growth

Ethanol tolerance

Gelatin liquefaction

Growth at $40^{\circ}$

Growth in yeast extract $(0.5 \%, w / v)$ medium

Milk peptonization

Ammonia production

Hydrogen sulphide production

Indole production

Nitrate reduction $†$

$\begin{array}{cc}\text { Parent } & \text { Mutant } \\ 5 \cdot 0-6 \cdot 0 & 7 \cdot 5-8 \cdot 5 \\ 4 \%,(v / v) & 10-12 \%,(v / v) \\ 0 / 3 * & 26 / 26 \\ 0 / 3 & 9 / 9 \\ 0 / 3 & 26 / 26 \\ 0 / 3 & 22 / 23 \\ 0 / 3 & 16 / 26 \\ 0 / 3 & 0 / 26 \\ 0 / 3 & 0 / 26 \\ 0 / 3 & 0 / 26\end{array}$

* Proportion of isolates tested which gave a positive reaction.

$\dagger \mathbf{A}$ positive test for nitrite after the addition of zinc dust indicated that nitrate was present. 
Additional experiments. The results of other experiments (Table 5) revealed that, unlike the parent cultures, the mutants (1) showed optimum growth at alkaline $\mathrm{pH}$ values, (2) tolerated $10-12 \%(\mathrm{v} / \mathrm{v})$ ethanol, (3) liquefied gelatin, (4) grew at $40^{\circ}$, and (5) grew in yeast-extract medium; in addition, most of the mutant isolates peptonized milk or produced ammonia. None of these abilities were shown by the parent cultures. Furthermore, none of the isolates tested (parent or mutant) produced hydrogen sulphide or indole or reduced nitrate. The adequacy of these test procedures was confirmed by using an organism which gave positive reactions under the conditions of test.

\section{DISCUSSION}

That the mutant isolates studied differ from the parent cultures in several respects is clear (Tables 2-5). Some of the differences observed between the parent and the mutant cultures were common to all parent and mutant isolates examined. For example, unlike the parent cultures, the mutants did not oxidize ethanol to acetic acid, showed optimum growth at alkaline $\mathrm{pH}$ values, were able to tolerate high concentrations of ethanol, liquefied gelatin, grew at $40^{\circ}$ and grew in yeast extract medium. In contrast to these associated changes, the differences observed in colony types (secondary colonies), nutritional requirements, oxidation of certain sugars and glycerol, probably represented independent changes since they occurred at random among the mutant cultures. It is also possible that some of the observed differences in nutritional requirements arose from an imbalance of amino acids supplied (cf. Gladstone, 1939).

In contrast with the results of Frateur (1950) is the fact that the oxidation of calcium lactate by the parent cultures was not demonstrated in the present work.

The failure to oxidize ethanol to acetic acid would appear to exclude the mutants as belonging to the genus Acetobacter (Bergey's Manual, 1948). Moreover, proteolytic activity is not found among members of this genus (Frateur, 1950). Apart from minor differences, the mutant organisms bear a resemblance to the description given for Pseudomonas geniculata in Bergey's Manual of Determinative Bacteriology. Our attempts to isolate, from samples of raw vinegar, bacteria which exhibit properties similar to those shown by the mutant organisms have been unsuccessful (results not given in the text).

One of us (R.S.) acknowledges the receipt of an Imperial Chemical Industries Research Fellowship.

\section{REFERENCES}

Bergey's Manual of Determinative Bacteriology (1948), 6th ed. Edited by BrEed, R. S., Murray, E. G. D. \& Hrtchens, A. P. Baltimore: The Williams and Wilkins Co.

Conn, H. J. (1949). Manual of Methods for Pure Culture Study of Bacteria. Geneva (New York, U.S.A.): Biotech. Publications. 
Creedy, A. E., JowetT, P. \& Walker, T. K. (1954). Formation of D-cellobiose from starch and from other substrates by an Acetobacter species. Chem. \& Ind. (Rev.), 73, 1279.

Frateur, J. (1950). Essai sur la systematique des acetobacters. La Cellule, 53, 287.

Gladstone, G. P. (1939). Inter-relationships between amino acids in the nutrition of B. anthracis. Brit. J. exp. Path. 20, 189.

MonoD, J. (1947). The phenomenon of enzymic adaptation and its bearing on problems of genetic and cellular differentiation. Growth, 11, 223.

Schramm, M. \& Hestrin, S. (1954). Factors affecting production of cellulose at the air-liquid interface of a culture of Acetobacter xylinum. J. gen. Microbiol. 11, 123.

Steel, R. \& Walker, T. K. (1957). Celluloseless mutants of certain Acetobacter species. J. gen. Microbiol. 17, 12.

Tosic, J. \& Walker, T. K. (1946). Procedure for the characterisation of the acetic acid bacteria. Chem. \& Ind. (Rev.), 65, 104.

Umbreit, W. W., Burris, R. H. \& Stauffer, J. F. (1945). Manometric Techniques and Tissue Metabolism. Minneapolis (Minnesota, U.S.A.): Burgess Publishing Co.

\section{EXPLANATION OF PLATE}

(Magnification, $\times 10$.)

Fig. 1. Colonies of cellulose-producing organisms.

Figs. 2-6. Colonies of celluloseless-mutant organisms (see text for details). 
Journal of General Microbiology, Vol. 17, No. 2
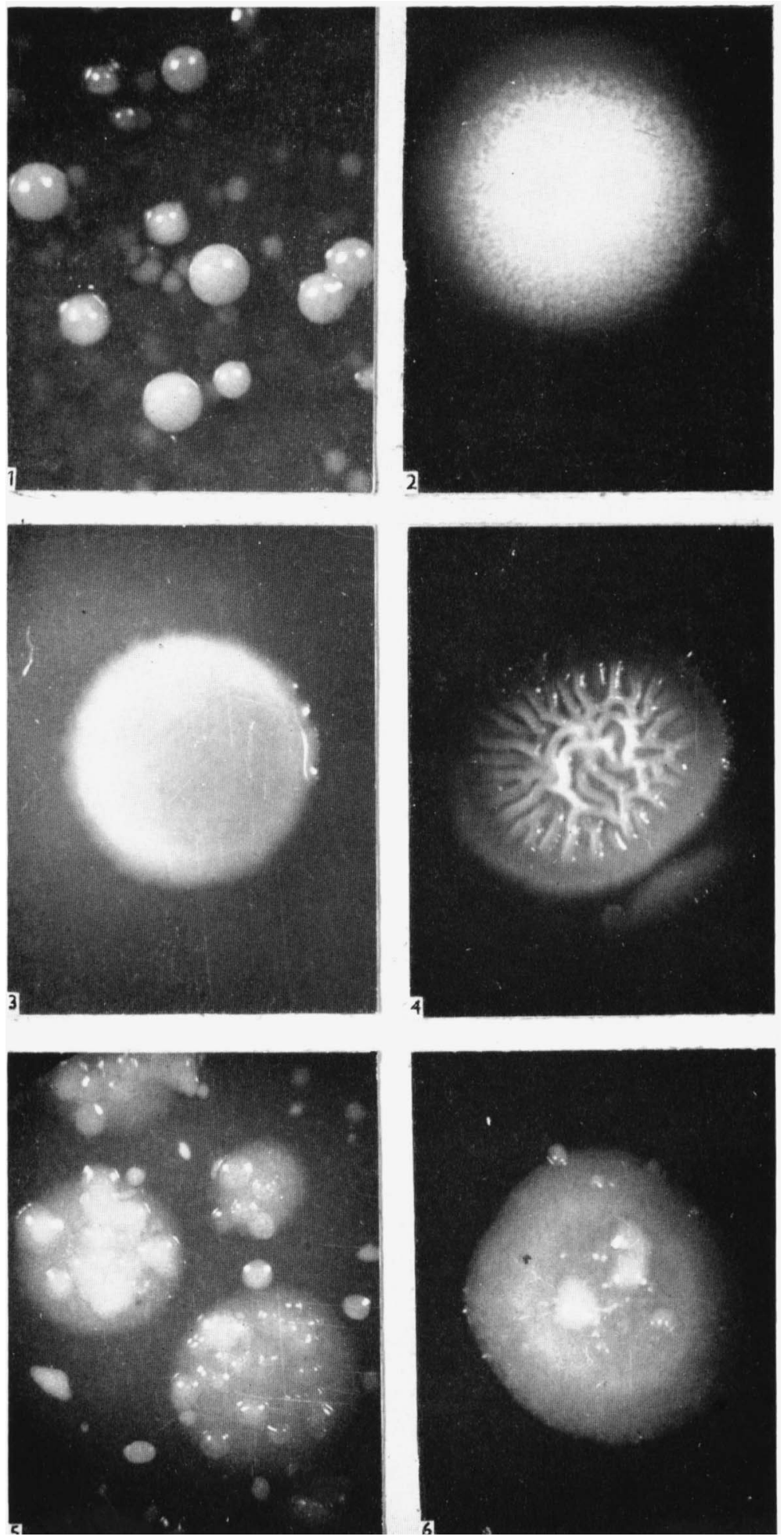

R. Steel \& T. K, Walker-Comparative studies of ACetobacter SpP. Plate 1

(Facing $p$. 452) 